\title{
Phase state and migration characteristics of natural gas in deep layer under the high temperature and pressure \\ ZHICHAO YU ${ }^{1}$; SHAOBO LIU ${ }^{2}$; XUESONG LU ${ }^{3}$
}

${ }^{1}$ PetroChina Exploration and Development Research Institute, Beijing 100083, China, yuzhichao@petrochins.com.cn

2 PetroChina Exploration and Development Research Institute, Beijing 100083, China, Isb@petrochina.com.cn

${ }^{3}$ PetroChina Exploration and Development Research Institute, Beijing 100083, China, luxs@petrochina.com.cn

Using the theoretical calculation and experimental analysis, the phase characteristics of natural gas under the high-temperature \& pressure conditions, including the density, viscosity, compression factor, solubility and interfacial tension, are studied in detail. Combined with the geological conditions, the vertical migration of natural gas in kela 2 gas field and the evolution of dissolved gas in the formation water are also discussed. The results show that under the conditions of high temperature \& pressure, most natural gas is under the condition of supercritical state, and its fluid property is between liquid and gas. It not only has density and solubility similar to liquid, but also has low viscosity and high diffusion coefficient similar to gas, which is very conducive to the migration and accumulation of natural gas. With the burial depth increasing, the density and viscosity of natural gas increased gradually, while the density and viscosity of formation water decreased. The solubility of natural gas in formation water increased. The interfacial tension between gas and water decreased. The decrease of gas-water interfacial tension indicates that the migration resistance of natural gas in deep layer is much smaller than that in shallow layer, which is very beneficial to the migration and preservation of natural gas. 